\title{
On the Boundedness Properties of Solutions to Set Control Differential Equations
}

\author{
Nguyen Dinh Phu ${ }^{1, *}$, Le Thanh Quang ${ }^{1}$, Lam Quoc Dung ${ }^{2}$ \\ ${ }^{1}$ Faculty of Mathematics and Computer Science,University of Science-VNU Ho Chi Minh City, Vietnam \\ ${ }^{2}$ Faculty of Economics and Commerce, Hoa Sen University, Ho Chi Minh City, Vietnam
}

\begin{abstract}
The set-valued differential equations (SDEs) are important parts of the set-valued analysis theory. It was investigeted by professor Lakshmikantham V., and many other authors (see[1]-[6],[8]-[10]). Beside that, we have to studied the problems of existence, comparis on and stability of set solutions to the set-valued control differential equations (SCDEs) (see[7],[11]-[16]). In this paper, we present the problems of boundedness for set solutions to the Set Control Differential Equations (SCDEs) by the Lyapunov-like functions and by admisible control- feedback.
\end{abstract}

Keywords Set Differential Equations (SDEs), Set Control Differential Equations (SCDEs)

\section{Introduction}

In the last 10 years, set-valued analys is is interesting with the new field of set differential equations (SDEs). There are many the authors are interesting in field of SDEs, for example, Lakshmikantham V., Gnana T., Kaleva O., Mohapatra R.,... Before we proceed to investigate our problems, let's note the following facts:

In[5], Prof.V. Lakshmikanthamand the other authors have studied the set differential equations (SDEs).

In[13] and [16], the authors have considered the set control differential equations (SCDEs), that is SDEs with set controls: $\mathrm{U}(\mathrm{t}) \in \mathrm{K}_{\mathrm{C}}\left(\mathrm{R}^{\mathrm{n}}\right)$ and have some important results on existence, stability.

$\operatorname{In}[11]$ the author has given many kinds of feedback $\mathrm{U}(\mathrm{t})=\mathrm{U}(\mathrm{X}(\mathrm{t})) \in \mathrm{K}_{\mathrm{C}}\left(\mathrm{R}^{\mathrm{n}}\right)$ for problem of global controllability.

In this paper, we present the boundedness of set solutions to SCDEs by the Liapunov-like functions and by feedback.

This paper is organized as follows: in section 2, we recall some basic concepts and notations which are useful in next sections. In section 3 we present the boundedness properties of set solutions to SCDEs and in the last section, we give the conclusion and acknowledgements.

\section{Preliminaries}

In[5], Prof. V. Lakshmikanthamand the other authors have

* Corresponding author:

ndphu_dhtn@yahoo.com.vn (Nguyen Dinh Phu)

Published online at http:/journal.sapub.org/am

Copyright (C) 2012 Scientific \& Academic Publishing. All Rights Reserved studied the set differential equations (SDEs). In this work the authors have considered the Hausdorff metric space as followings:

Let $\mathrm{K}_{\mathrm{C}}\left(\mathrm{R}^{\mathrm{n}}\right)$ denote the collection of all nonempty convex subsets of $R^{n}$. Given $A, B \in K_{C}\left(R^{n}\right)$ - the Hausdorff distance between $\mathrm{A}$ and $\mathrm{B}$ is defined by

$$
d_{H}[A, B]=\max \left\{\sup _{a \in A} d(a, B), \sup _{b \in B} d(b, A)\right\}
$$

We define the magnitude of a nonempty subset of $A$

$$
\mathrm{d}_{\mathrm{H}}\left[\mathrm{A}, \theta^{\mathrm{n}}\right]=\|\mathrm{A}\|
$$

We define the magnitude of a nonempty subset of $\mathrm{A}$ :

$$
D\left[A, \theta^{n}\right]=\|A\|=\sup \left\{\|a\|_{R^{n}}, a \in A\right\}
$$

where $\theta^{n}$ is the zero element of $\mathrm{R}^{\mathrm{n}}$ which is regarded as a one point set. $\|A\|=D\left[A, \theta^{n}\right]$ - norm in $K_{C}\left(R^{n}\right)$ is finite when the supremum in (1) is attained with $A \in K_{C}\left(R^{n}\right)$. The set $\mathrm{K}_{\mathrm{C}}\left(\mathrm{R}^{\mathrm{n}}\right)$, with the metric $\mathrm{D}$ defined above, is a complete metric space. It has been proven that $\mathrm{K}_{\mathrm{C}}\left(\mathrm{R}^{\mathrm{n}}\right)$ becomes a semilinear metric space which can be embedded as a complete cone into a corresponding Banach space, if it is equipped with the natural algebraic operations of addition and nonnegative scalar mult iplication.

Let $A, B \in K_{C}\left(R^{n}\right)$ if there exists a set $C \in K_{C}\left(R^{n}\right)$ such that $\mathrm{A}=\mathrm{B}+\mathrm{C}$, then $\mathrm{C}$ is called the Hausdorff difference (the geometric difference) of the sets $\mathrm{A}$ and $\mathrm{B}$ and is denoted by the symbol $\mathrm{A}-\mathrm{B}$. The mapping

$\mathrm{X}: \mathrm{R}_{+} \supset \mathrm{I}=\left[\mathrm{t}_{0}, \mathrm{~T}\right] \rightarrow \mathrm{K}_{\mathrm{C}}\left(\mathrm{R}^{\mathrm{n}}\right)$ is said to have a Hukuhara derivative $D_{H} X(\tau)$ at a point $\tau \in I$, if

$$
\begin{aligned}
& \lim _{h \rightarrow 0^{+}} h^{-1}[X(\tau+h)-X(\tau)] \text { and } \lim _{h \rightarrow 0^{+}} h^{-1}[X(\tau)-X(\tau-h)] \\
& \text { exist in the topology of } K_{C}\left(R^{h}\right) \text { and are equal to } D_{H} X(\tau) .
\end{aligned}
$$


By embedding $\mathrm{K}_{\mathrm{C}}\left(\mathrm{R}^{\mathrm{n}}\right)$ as a complete cone in a corresponding Banach space and taking into account the result on the differentiation of Bochner integral, we find that if $\mathrm{X}(\mathrm{t})=\mathrm{X}_{0}+\int_{\mathrm{t}_{0}}^{\mathrm{t}} \Phi(\mathrm{s}) \mathrm{ds}, \mathrm{X}_{0} \in \mathrm{K}_{\mathrm{c}}\left(\mathrm{R}^{\mathrm{n}}\right)$,

where $\Phi:\left[\mathrm{t}_{0}, \mathrm{~T}\right] \rightarrow \mathrm{K}_{\mathrm{C}}\left(\mathrm{R}^{\mathrm{n}}\right)$ is integrable in the sence of Bochner, then $\mathrm{D}_{\mathrm{H}} \mathrm{X}(\tau)$ exists and the equality

$$
\mathrm{D}_{\mathrm{H}} \mathrm{X}(\tau)=\Phi(\tau)
$$

\section{Main Results}

Let's consider the set control differential equations (SCDEs):

$$
D_{H} X(t)=F(t, X(t), U(t))
$$

where

$$
\mathrm{X}\left(\mathrm{t}_{0}\right)=\mathrm{X}_{0} \in \mathrm{K}_{\mathrm{C}}\left(\mathrm{R}^{\mathrm{n}}\right), \mathrm{F} \in\left[\mathrm{I} \times \mathrm{K}_{\mathrm{C}}\left(\mathrm{R}^{\mathrm{n}}\right) \times \mathrm{K}_{\mathrm{C}}\left(\mathrm{R}^{\mathrm{d}}\right), \mathrm{K}_{\mathrm{C}}\left(\mathrm{R}^{\mathrm{n}}\right)\right]
$$

$\mathrm{t} \in\left[\mathrm{t}_{0}, \mathrm{~T}\right]$, state $\mathrm{X}(\mathrm{t}) \in \mathrm{K}_{\mathrm{C}}\left(\mathrm{R}^{\mathrm{n}}\right)$ and control $\mathrm{U}(\mathrm{t}) \in \mathrm{K}_{\mathrm{C}}\left(\mathrm{R}^{\mathrm{d}}\right)$ If

$\mathrm{U}:\left[\mathrm{t}_{0}, \mathrm{~T}\right] \rightarrow \mathrm{K}_{\mathrm{C}}\left(\mathrm{R}^{\mathrm{d}}\right)$ integrable, then it is called an admissible control. Let $\mathrm{U}$ be a set of all admissible controls The mapping $\mathrm{X}(\mathrm{t}) \in \mathrm{C}^{1}\left([0, \mathrm{~T}], \mathrm{K}_{\mathrm{C}}\left(\mathrm{R}^{\mathrm{n}}\right)\right)$ is said to be a solution of SCDES (3) on $t \in\left[t_{0}, T\right]$ iff it satisfies SCDEs on $t \in\left[t_{0}, T\right]$ and is the symbolic representation of the following Hukuhara integral expression:

$$
X(t)=X_{0}+\int_{0}^{t} F(s, X(s), U(s)) d s
$$

Definition 3.1. The set solution $\mathrm{X}(\mathrm{t})$ of SCDEs (3) is said to be:

$\mathrm{a} /$ (B)- bounded on $\mathrm{t} \in\left[\mathrm{t}_{0}, \mathrm{~T}\right]$, if there exists the constant $M>0$ such that, by (4) we have $\|X(t)\| \leq M$, for all $\mathrm{t} \in\left[\mathrm{t}_{0}, \mathrm{~T}\right]$.

$\mathrm{b} /(\mathrm{EB})$ - Exponent bounded on $\mathrm{t} \in\left[\mathrm{t}_{0}, \mathrm{~T}\right]$, if there exist the constants $\beta, M \in \mathrm{R}^{+}$such that the supper distance: $\|\mathrm{X}(\mathrm{t})\| \leq \mathrm{M} \cdot \mathrm{e}^{\left[-\beta\left(\mathrm{t}-\mathrm{t}_{0}\right)\right]}, \forall \mathrm{t} \in[0, \mathrm{~T}]$.

Assume that $\mathrm{F}: \mathrm{R}^{\mathrm{n}} \times \mathrm{K}_{\mathrm{C}}\left(\mathrm{R}^{\mathrm{n}}\right) \times \mathrm{K}_{\mathrm{C}}\left(\mathrm{R}^{\mathrm{d}}\right) \rightarrow \mathrm{K}_{\mathrm{C}}\left(\mathrm{R}^{\mathrm{n}}\right)$

satis fies the followings:

(F1). there exists a constant $\mathrm{L}>0$ such that

$$
\begin{aligned}
& \mathrm{D}[\mathrm{F}(\mathrm{t}, \mathrm{A}, \mathrm{B}), \mathrm{F}(\mathrm{t}, \overline{\mathrm{A}}, \overline{\mathrm{B}})] \leq \mathrm{L}(\mathrm{D}[\mathrm{A}, \overline{\mathrm{A}}]+\mathrm{D}[\mathrm{B}, \overline{\mathrm{B}}]), \\
& \forall \mathrm{A}, \overline{\mathrm{A}} \in \mathrm{K}_{\mathrm{C}}\left(\mathrm{R}^{\mathrm{n}}\right), \mathrm{B}, \overline{\mathrm{B}} \in \mathrm{K}_{\mathrm{C}}\left(\mathrm{R}^{\mathrm{d}}\right) ;
\end{aligned}
$$

(F2). there exists constant $\mathrm{C}>0$ such that

$\left.\mathrm{D}\left[\mathrm{F}(\mathrm{t}, \mathrm{A}, \mathrm{B}), \theta^{\mathrm{n}}\right]\right) \leq \mathrm{C}\left(1+\mathrm{D}\left[\mathrm{A}, \theta^{\mathrm{n}}\right]+\mathrm{D}\left[\mathrm{B}, \theta^{\mathrm{d}}\right]\right)$;

(U1). $\int_{\mathrm{t}_{0}}^{\mathrm{t}}\|\mathrm{U}(\mathrm{s})\| \mathrm{ds}<+\infty$.

Theorem 3.1. Let $\mathrm{F} \in \mathrm{K}_{\mathrm{C}}\left(\mathrm{R}^{\mathrm{n}}\right)$ satisfies hypotheses (F1)-(F2) and $U$ satisfies (U1), then SCDEs (3) has unique B- bounded set solutions $X(t) \in K_{C}\left(R^{n}\right)$.

Proof. We have to prove that: a) By (F1), there exists the set solutions, which is represented as (4).

b) Uniqueness of $X(t)$. As sume that the other set solutions $\mathrm{Y}(\mathrm{t}) \in \mathrm{K}_{\mathrm{C}}(\mathrm{R})$ such that $\mathrm{X}\left(\mathrm{t}_{0}\right)=\mathrm{Y}\left(\mathrm{t}_{0}\right)$, then $\mathrm{X}(\mathrm{t}) \equiv \mathrm{Y}(\mathrm{t}), \forall \mathrm{t} \geq \mathrm{t}_{0}$ in force (F2).

c) A boundedness property of set solution $X(t)$ that means there exists $M>0$ such that $\|X(t)\| \leq M$ for all $t \in\left[t_{0}, T\right]$.

We estimate $\|\mathrm{X}(\mathrm{t})\|$ by $(4)$ and $(\mathrm{F} 2)$ :

$$
\begin{gathered}
\|X(t)\|=D\left[X(t), \theta^{n}\right] \leq D\left[X_{0}+\int_{0}^{T} F(s, X(s), U(s)) d s, \theta^{n}\right] \\
\|X(t)\|=D\left[X(t), \theta^{n}\right] \leq D\left[X_{0}+\int_{0}^{T} C \cdot[1+\|X(s)\|+\|U(s)\|] d s, \theta^{n}\right]
\end{gathered}
$$

Putting $1(t)=\|X(t)\|, \quad k(t)=(1+|\lambda(t)|)$ and $a=\left\|X_{0}\right\|+C T$, we have $\left.1(\mathrm{t}) \leq \mathrm{a} \cdot+\mathrm{C} \int_{\mathrm{t}_{0}}^{\mathrm{t}}[1(\mathrm{~s}) \cdot \mathrm{k}(\mathrm{s}))\right] \mathrm{ds}$.

This Gronwall's inequality implies that $1(t) \leq a \cdot \exp \left[2\left(t-t_{0}\right)\right]$

Choosing $M \geq \frac{C T \exp \left[2\left(T-t_{0}\right)\right]}{1-\exp \left[2\left(T-t_{0}\right)\right]}$, we have $1(t) \leq M$ for all $\mathrm{t} \in\left[\mathrm{t}_{0}, \mathrm{~T}\right]$

Theorem 3.2. Let $X_{0} \in K_{C}\left(R^{n}\right), \quad D\left[X_{0}, \theta^{n}\right] \leqslant M_{1}$ and $\int_{0}^{\mathrm{t}} \mathrm{D}\left[\mathrm{F}\left(\mathrm{s}, \theta^{\mathrm{n}}, \theta^{\mathrm{d}}\right), \theta^{\mathrm{n}}\right] \mathrm{ds} \leq \mathrm{M}_{2}$ and by contraction feedback $\mathrm{U}(\mathrm{t})=\mathrm{h}(\mathrm{t}) \mathrm{X}(\mathrm{t})$ and $0<\mathrm{h}(\mathrm{t}) \leqslant 1$, then SCDEs (3) has the unique (B)- bounded solution in $\mathrm{K}_{\mathrm{C}}\left(\mathrm{R}^{\mathrm{n}}\right)$.

Proof. (a) Problems of existence and uniqueness are clear.

(b) Problem of (B)- bounded are proved by integral expression (4) followings:

$$
\mathrm{D}\left[\mathrm{X}_{0}, \theta^{\mathrm{n}}\right] \leqslant \mathrm{M}_{1},
$$

and

$$
\begin{aligned}
& \|X(t)\|=D\left[X(t), \theta^{\mathrm{n}}\right] \leq \mathrm{D}\left[\mathrm{X}_{0}+\int_{0}^{\mathrm{T}} \mathrm{F}(\mathrm{s}, \mathrm{X}(\mathrm{s}), \mathrm{U}(\mathrm{s})) \mathrm{ds}, \theta^{\mathrm{n}}\right] \\
& \quad \leqslant \mathrm{M}_{1}+2 \mathrm{D}\left[\mathrm{X}(\cdot), \theta^{\mathrm{n}}\right]+2 \mathrm{C}+\mathrm{M}_{2}+2 \int_{0}^{\mathrm{t}} \mathrm{D}\left[\mathrm{X}(\mathrm{s}), \theta^{\mathrm{n}}\right] \mathrm{ds}
\end{aligned}
$$

Using Gronwall's inequality, we infer

$$
\mathrm{D}\left[\mathrm{x}(\mathrm{t}), \theta^{\mathrm{n}}\right] \leqslant\left[\mathrm{M}+2 \mathrm{D}\left[\mathrm{X}(\cdot), \theta^{\mathrm{n}}\right]\right] \exp (2 \mathrm{~T}),
$$

where $M=M_{1}+M_{2}+2 C$, we obtain $D\left(X(t), \theta^{n}\right) \leqslant M$.

Next, we present some results about (B), (EB) of solutions in $\mathrm{K}_{\mathrm{C}}\left(\mathrm{R}^{\mathrm{n}}\right)$ with using the Lyapunov-like functions.

Theorem 3.3. Assume that the positive Lyapunov - like function $\mathrm{V} \in \mathrm{C}\left[\mathrm{R}_{+} \times \mathrm{K}_{\mathrm{C}}\left(\mathrm{R}^{\mathrm{n}}\right) \times \mathrm{K}_{\mathrm{C}}\left(\mathrm{R}^{\mathrm{d}}\right), \mathrm{R}_{+}\right]$which satisfies the following conditions:

(i) $\begin{aligned} & |\mathrm{V}(\mathrm{t}, \mathrm{X}(\mathrm{t}), \mathrm{U}(\mathrm{t}))-\mathrm{V}(\mathrm{t}, \overline{\mathrm{X}}(\mathrm{t}), \overline{\mathrm{U}}(\mathrm{t}))| \leq \\ & \mathrm{L}(\mathrm{D}[\mathrm{X}(\mathrm{t}), \overline{\mathrm{X}}(\mathrm{t})]+\mathrm{D}[\mathrm{U}(\mathrm{t}), \overline{\mathrm{U}}(\mathrm{t})])\end{aligned}$, where $\quad \mathrm{L} \quad$ is bounded Lipschitz constant, for all

$\mathrm{X}(\mathrm{t}), \overline{\mathrm{X}}(\mathrm{t}) \in \mathrm{K}_{\mathrm{C}}\left(\mathrm{R}^{\mathrm{n}}\right), \mathrm{u}(\mathrm{t}), \overline{\mathrm{u}}(\mathrm{t}) \in \mathrm{K}_{\mathrm{C}}\left(\mathrm{R}^{\mathrm{d}}\right)$ and $\mathrm{t} \in \mathrm{R}_{+}$;

(ii) $\mathrm{b}\left(\mathrm{D}\left[\mathrm{x}(\mathrm{t}), \theta^{\mathrm{nN}}\right]\right) \leq \mathrm{V}(\mathrm{t}, \mathrm{X}(\mathrm{t}), \mathrm{U}(\mathrm{t})) \leq \mathrm{a}\left(\mathrm{t}, \mathrm{D}\left[\mathrm{X}(\mathrm{t}), \theta^{\mathrm{n}}\right]\right)$, for $(\mathrm{t}, \mathrm{X}, \mathrm{U}) \in \mathrm{R}_{+} \times \mathrm{K}_{\mathrm{C}}\left(\mathrm{R}^{\mathrm{n}}\right) \times \mathrm{K}_{\mathrm{C}}\left(\mathrm{R}^{\mathrm{d}}\right)$, where $\mathrm{b}(),. \mathrm{a}(\mathrm{t},$.$) are$ increasing functions; 
(iii)

$\mathrm{D}^{+} \mathrm{V}(\mathrm{t}, \mathrm{X}(\mathrm{t}), \mathrm{U}(\mathrm{t})) \equiv$

$\lim _{\tau \rightarrow 0^{+}} \sup \frac{1}{\tau}\{\mathrm{V}(\mathrm{t}+\tau, \mathrm{X}(\mathrm{t})+\tau \mathrm{f}(\mathrm{t}, \mathrm{X}(\mathrm{t}), \mathrm{U}(\mathrm{t})))-\mathrm{V}(\mathrm{t}, \mathrm{X}(\mathrm{t}), \mathrm{U}(\mathrm{t}))\}$

$\leq \mathrm{g}(\mathrm{t}, \mathrm{V}(\mathrm{t}, \mathrm{X}(\mathrm{t}), \mathrm{U}(\mathrm{t}))))$,

where $g \in C\left[R_{+}^{2}, R\right], g(t, 0)=0$ for all

$\mathrm{X}(\mathrm{t}) \in \mathrm{K}_{\mathrm{C}}\left(\mathrm{R}^{\mathrm{n}}\right), \mathrm{U}(\mathrm{t}) \in \mathrm{K}_{\mathrm{C}}\left(\mathrm{R}^{\mathrm{d}}\right)$ and $\mathrm{t} \in \mathrm{R}_{+}$, we have the following affirmations:

$\mathrm{a} /$ If $\mathrm{g}(\mathrm{t}, \mathrm{V}(\mathrm{t}, \mathrm{X}(\mathrm{t}), \mathrm{U}(\mathrm{t}))) \leq 0$, then a set solution

$\mathrm{X}(\mathrm{t}) \in \mathrm{K}_{\mathrm{C}}\left(\mathrm{R}^{\mathrm{n}}\right)$ of SCDEs (3) is (B)-bounded.

$\mathrm{b} /$ If $\mathrm{g}(\mathrm{t}, \mathrm{V}(\mathrm{t}, \mathrm{X}(\mathrm{t}), \mathrm{U}(\mathrm{t})))<0$,

(or if $\mathrm{g}(\mathrm{t}, \mathrm{V}(\mathrm{t}, \mathrm{X}(\mathrm{t}), \mathrm{U}(\mathrm{t})))<-\beta \mathrm{V}, \forall \mathrm{t} \geq \mathrm{t}_{0}$ ) then a set solution $\mathrm{X}(\mathrm{t}) \in \mathrm{K}_{\mathrm{C}}\left(\mathrm{R}^{\mathrm{n}}\right)$ of SCDEs (3) is (EB)-bounded.

Proof. Setting the function $m(t)=V(t, X(t), U(t))$, we have $\mathrm{D}^{+} \mathrm{m}(\mathrm{t})=\mathrm{D}^{+} \mathrm{V}(\mathrm{t}, \mathrm{X}(\mathrm{t}), \mathrm{U}(\mathrm{t}))=$

$$
\lim _{\tau \rightarrow 0^{+}} \sup \frac{1}{\tau}\{\mathrm{V}(\mathrm{t}+\tau, \mathrm{X}(\mathrm{t})+\tau \mathrm{f}(\mathrm{t}, \mathrm{X}(\mathrm{t}), \mathrm{U}(\mathrm{t})))-\mathrm{V}(\mathrm{t}, \mathrm{X}(\mathrm{t}), \mathrm{U}(\mathrm{t}))\}
$$

$\leq \mathrm{g}(\mathrm{t}, \mathrm{V}(\mathrm{t}, \mathrm{X}(\mathrm{t}), \mathrm{U}(\mathrm{t})))$, so $\mathrm{D}^{+} \mathrm{m}(\mathrm{t}) \leq \mathrm{g}(\mathrm{t}, \mathrm{m}(\mathrm{t}))$, implies that $\mathrm{m}\left(\mathrm{t}_{0}\right) \leq \omega_{0}$. Since $\mathrm{m}(\mathrm{t}) \leq \mathrm{r}\left(\mathrm{t}_{0}, \omega_{0}, \mathrm{t}\right)$ where $\mathrm{r}\left(\mathrm{t}_{0}, \omega_{0}, \mathrm{t}\right)$ is maximal solution of ODE:

$$
\frac{d \omega}{d t}=g(t, \omega)
$$

then $\mathrm{V}(\mathrm{t}, \mathrm{X}(\mathrm{t}), \mathrm{U}(\mathrm{t})) \leq \mathrm{V}(\cdot, \mathrm{X}(\cdot), \mathrm{U}(\cdot)) \leq \mathrm{V}\left(\mathrm{t}_{0}, \mathrm{X}_{0}, \mathrm{U}_{0}\right)$ for all $0 \leq \mathrm{t} \leq \mathrm{T}$

- Let $0<\varepsilon<\mathrm{r}, \mathrm{t}_{0} \in \mathrm{R}_{+}$, be given. Choose a $\delta=\delta\left(\mathrm{t}_{0}, \varepsilon\right)$ such that $\mathrm{a}\left(\mathrm{t}_{0}, \delta\right)<\mathrm{b}(\varepsilon)$. We claim that with this $\delta$ then (B)bounded solution. If it's not true, there exists solution $X(t)$ of SCDEs (3) and $t^{\prime}>t$, such that $D\left[X\left(t^{\prime}\right), \theta^{n}\right]=\beta$ and $\mathrm{D}\left[\mathrm{X}(\mathrm{t}), \theta^{\mathrm{n}}\right] \leq \varepsilon<\mathrm{r}$, where $\beta>\varepsilon$ for all $\mathrm{t}<\mathrm{t}^{\prime} \leq \mathrm{T}$.

Wherever $\mathrm{D}\left[\mathrm{X}_{0}, \theta^{\mathrm{n}}\right]<\delta$, because

$\mathrm{V}\left(\mathrm{t}, \mathrm{X}\left(\mathrm{t}^{\prime}\right), \mathrm{U}\left(\mathrm{t}^{\prime}\right)\right) \leq \mathrm{V}(\mathrm{t}, \mathrm{X}(\mathrm{t}), \mathrm{U}(\mathrm{t})) \leq$

$$
\mathrm{V}(\cdot, \mathrm{X}(\cdot), \mathrm{U}(\cdot)) \leq \mathrm{V}\left(\mathrm{t}_{0}, \mathrm{X}_{0}, \mathrm{U}_{0}\right)
$$

for all $0 \leq \mathrm{t}<\mathrm{t}^{\prime} \leq \mathrm{T}$

then

$\mathrm{b}(\beta)=\mathrm{b}\left(\mathrm{D}\left[\mathrm{X}\left(\mathrm{t}^{\prime}\right), \theta^{\mathrm{n}}\right]\right) \leq \mathrm{V}\left(\mathrm{t}^{\prime}, \mathrm{X}\left(\mathrm{t}^{\prime}\right), \mathrm{U}\left(\mathrm{t}^{\prime}\right)\right) \leq$

$\mathrm{V}(\mathrm{t}, \mathrm{X}(\mathrm{t}), \mathrm{U}(\mathrm{t})) \leq \mathrm{V}\left(\mathrm{t}_{0}, \mathrm{X}_{0}, \mathrm{U}_{0}\right) \leq \mathrm{a}\left(\mathrm{t}_{0}, \mathrm{D}\left[\mathrm{X}_{0}, \theta^{\mathrm{n}}\right]\right) \leq \mathrm{a}\left(\mathrm{t}_{0}, \delta\right)<\mathrm{b}(\varepsilon)$

by $b($.$) is a increasing function, therefore this contradiction$ proves that (B)-bounded solution .

- In the case, if $\mathrm{g}(\mathrm{t}, \mathrm{V}(\mathrm{t}, \mathrm{X}(\mathrm{t}), \mathrm{U}(\mathrm{t})))<0$ (or $\mathrm{D}^{+} \mathrm{V}(\mathrm{t}, \mathrm{X}(\mathrm{t}), \mathrm{U}(\mathrm{t}))<-\beta \mathrm{V}(\mathrm{t}, \mathrm{X}(\mathrm{t}), \mathrm{U}(\mathrm{t})) \quad$ ) then we have $\mathrm{V}(\mathrm{t}, \mathrm{X}(\mathrm{t}), \mathrm{U}(\mathrm{t})) \leq \mathrm{V}\left(\mathrm{t}_{0}, \mathrm{X}_{0}, \mathrm{U}_{0}\right)$ for all $0 \leq \mathrm{t} \leq \mathrm{T}$.

If $\quad \mathrm{D}^{+} \mathrm{V}(\mathrm{t}, \mathrm{X}(\mathrm{t}), \mathrm{U}(\mathrm{t}))<-\beta \mathrm{V}(\mathrm{t}, \mathrm{X}(\mathrm{t}), \mathrm{U}(\mathrm{t}))$

then

$\mathrm{V}(\mathrm{t}, \mathrm{X}(\mathrm{t}), \mathrm{U}(\mathrm{t})) \leq \mathrm{V}\left(\mathrm{t}_{0}, \mathrm{X}_{0}, \mathrm{U}_{0}\right) \cdot \mathrm{e}^{\left[-\beta\left(\mathrm{t}-\mathrm{t}_{0}\right)\right]}, \forall 0 \leq \mathrm{t} \leq \mathrm{T}$ and if (EB)

is not true, given $\varepsilon_{0}$, we choose

$$
\begin{aligned}
& \mathrm{T}=\mathrm{T}\left(\mathrm{t}_{0}, \varepsilon_{0}\right)=\frac{1}{\beta} \ln \frac{\mathrm{a}\left(\mathrm{t}_{0}, \delta\right)}{\mathrm{b}\left(\varepsilon_{0}\right)}+1 \text { then } \\
& \mathrm{b}\left(\mathrm{D}\left[\mathrm{x}(\mathrm{t}), \theta^{\mathrm{nN}}\right]\right) \leq \mathrm{V}(\mathrm{t}, \mathrm{X}(\mathrm{t}), \mathrm{U}(\mathrm{t})) \leq \mathrm{a}\left(\mathrm{t}_{0}, \delta\right) \cdot \mathrm{e}^{\left[-\beta\left(\mathrm{t}-\mathrm{t}_{0}\right)\right]}<\mathrm{b}(\varepsilon),
\end{aligned}
$$

for all $0 \leq \mathrm{t} \leq \mathrm{T}$, this contradiction proves that the fuzzy set solution $X(t) \in K_{C}\left(R^{n}\right)$ is $(E B)$.

Definition 3.2. The set solutions of SCDEs (3) are said to be:

a/ (B1)- equi - bounded, if for any $\alpha>0$ and $t_{0} \in R_{+}$, there exists a $\quad \beta=\beta\left(\mathrm{t}_{0}, \alpha\right)>0 \quad$ such that $\left\|\mathrm{X}_{0}\right\|<\alpha$ implies $\|\mathrm{X}(\mathrm{t})\|<\beta, \mathrm{t} \geq \mathrm{t}_{0}$. $\mathrm{b} /(\mathrm{B} 2)$ - uniform - bounded, if $\beta$ in (B1) does not depend on $t_{0}$.

Theorem 3.4. Assume that the Lyapunov-like function $\mathrm{V} \in \mathrm{C}\left[\mathrm{R}_{+} \times \mathrm{K}_{\mathrm{C}}\left(\mathrm{R}^{\mathrm{n}}\right) \times \mathrm{U}, \mathrm{R}_{+}\right]$and feedback $\mathrm{U}(\mathrm{t})=\lambda(\mathrm{t}) \mathrm{X}(\mathrm{t})$ satis fy the following conditions:

(i) $\begin{aligned} & |\mathrm{V}(\mathrm{t}, \overline{\mathrm{X}}(\mathrm{t}), \overline{\mathrm{U}}(\mathrm{t}))-\mathrm{V}(\mathrm{t}, \mathrm{X}(\mathrm{t}), \mathrm{U}(\mathrm{t}))| \leq \\ & \mathrm{L}(\mathrm{D}[\overline{\mathrm{X}}(\mathrm{t}), \mathrm{X}(\mathrm{t})]-\mathrm{D}[\overline{\mathrm{U}}(\mathrm{t}), \mathrm{U}(\mathrm{t})])\end{aligned}$

where $\mathrm{L}$ is bounded Lipschitz constant, for all $\overline{\mathrm{X}}, \mathrm{X} \in \mathrm{K}_{\mathrm{C}}\left(\mathrm{R}^{\mathrm{n}}\right) ; \overline{\mathrm{U}}, \mathrm{U} \in \mathrm{U}$ and $\mathrm{t} \in \mathrm{R}_{+}$;

(ii) add condition $\mathrm{D}^{+} \mathrm{V}(\mathrm{t}, \mathrm{X}(\mathrm{t}), \mathrm{U}(\mathrm{t})) \equiv$

$$
\begin{aligned}
& \limsup _{\mathrm{h} \rightarrow 0^{+}} \frac{1}{\mathrm{~h}}\{\mathrm{~V}(\mathrm{t}+\mathrm{h}, \mathrm{X}(\mathrm{t})+\mathrm{hF}(\mathrm{t}, \mathrm{X}(\mathrm{t}), \mathrm{U}(\mathrm{t})), \mathrm{U}(\mathrm{t}) \\
&\left.+\mathrm{h}\left[\lambda(\mathrm{t}) \mathrm{F}(\mathrm{t}, \mathrm{X}(\mathrm{t}), \mathrm{U}(\mathrm{t}))+\lambda^{\prime}(\mathrm{t}) \mathrm{X}(\mathrm{t})\right]\right\}
\end{aligned}
$$

$-\mathrm{V}(\mathrm{t}, \mathrm{X}(\mathrm{t}), \mathrm{U}(\mathrm{t}))\} \leq \mathrm{g}(\mathrm{t}, \mathrm{V}(\mathrm{t}, \mathrm{X}(\mathrm{t}), \mathrm{U}(\mathrm{t})))$

where $g \in C\left[R_{+}^{2}, R\right]$ and $t \in R_{+}, X(t) \in K_{C}\left(R^{n}\right), U \in U$.

If $\mathrm{X}(\mathrm{t})=\mathrm{X}\left(\mathrm{t}, \mathrm{t}_{0}, \mathrm{X}_{0}, \mathrm{U}_{0}\right)$ is any solution of SCDEs (3) existing on $\left[\mathrm{t}_{0}, \mathrm{~T}\right)$ such that $\mathrm{V}\left(\mathrm{t}_{0}, \mathrm{X}_{0}, \mathrm{U}_{0}\right) \leq \omega_{0}$, then we have $\mathrm{V}(\mathrm{t}, \mathrm{X}(\mathrm{t}), \mathrm{U}(\mathrm{t})) \leq \mathrm{r}\left(\mathrm{t}, \mathrm{t}_{0}, \omega_{0}\right)$, where $\mathrm{r}\left(\mathrm{t}, \mathrm{t}_{0}, \omega_{0}\right)$ is a maximal solution of ordinary differential equation (ODE) (5)

$$
\frac{\mathrm{d} \omega}{\mathrm{dt}}=\mathrm{g}(\mathrm{t}, \omega), \quad \omega\left(\mathrm{t}_{0}\right)=\omega_{0} \geq 0 .
$$

Proof. Let $\mathrm{X}(\mathrm{t})=\mathrm{X}\left(\mathrm{t}, \mathrm{t}_{0}, \mathrm{X}_{0}, \mathrm{U}_{0}\right)$ is any solution of SCDEs (3) existing on $\left[t_{0}, T\right)$.

Define $m(t)=V(t, X(t), U(t))$ so that

$\mathrm{m}\left(\mathrm{t}_{0}\right)=\mathrm{V}\left(\mathrm{t}_{0}, \mathrm{X}_{0}, \mathrm{U}_{0}\right) \leq \mathrm{w}_{0}$. Now, for small $\mathrm{h}>0$, by our assumption it follows that

$$
\begin{gathered}
\mathrm{m}(\mathrm{t}+\mathrm{h})-\mathrm{m}(\mathrm{t})= \\
\mathrm{V}(\mathrm{t}+\mathrm{h}, \mathrm{X}(\mathrm{t}+\mathrm{h}), \mathrm{U}(\mathrm{t}+\mathrm{h}))-\mathrm{V}(\mathrm{t}, \mathrm{X}(\mathrm{t}), \mathrm{U}(\mathrm{t}))= \\
\mathrm{V}(\mathrm{t}+\mathrm{h}, \mathrm{X}(\mathrm{t}+\mathrm{h}), \mathrm{U}(\mathrm{t}+\mathrm{h})) \\
-\mathrm{V}(\mathrm{t}+\mathrm{h}, \mathrm{X}(\mathrm{t})+\mathrm{hF}(\mathrm{t}, \mathrm{X}(\mathrm{t}), \mathrm{U}(\mathrm{t})), \mathrm{U}(\mathrm{t})+ \\
\left.\mathrm{h}\left[\lambda(\mathrm{t}) \mathrm{F}(\mathrm{t}, \mathrm{X}(\mathrm{t}), \mathrm{U}(\mathrm{t}))+\lambda^{\prime}(\mathrm{t}) \mathrm{X}(\mathrm{t})\right]\right) \\
+\mathrm{V}(\mathrm{t}+\mathrm{h}, \mathrm{X}(\mathrm{t})+\mathrm{hF}(\mathrm{t}, \mathrm{X}(\mathrm{t}), \mathrm{U}(\mathrm{t})), \mathrm{U}(\mathrm{t})+ \\
\left.\mathrm{h}\left[\lambda(\mathrm{t}) \mathrm{F}(\mathrm{t}, \mathrm{X}(\mathrm{t}), \mathrm{U}(\mathrm{t}))+\lambda^{\prime}(\mathrm{t}) \mathrm{X}(\mathrm{t})\right]\right) \\
-\mathrm{V}(\mathrm{t}, \mathrm{X}(\mathrm{t}), \mathrm{U}(\mathrm{t})) \\
\leq \mathrm{L}[\mathrm{D}[\mathrm{X}(\mathrm{t}+\mathrm{h}), \mathrm{X}(\mathrm{t})+\mathrm{hF}(\mathrm{t}, \mathrm{X}(\mathrm{t}), \mathrm{U}(\mathrm{t}))] \\
-\mathrm{D}\left[\mathrm{U}(\mathrm{t}+\mathrm{h}), \mathrm{U}(\mathrm{t})+\mathrm{h}\left[\lambda(\mathrm{t}) \mathrm{F}(\mathrm{t}, \mathrm{X}(\mathrm{t}), \mathrm{U}(\mathrm{t}))+\lambda^{\prime}(\mathrm{t}) \mathrm{X}(\mathrm{t})\right]\right] \\
+\mathrm{V}(\mathrm{t}+\mathrm{h}, \mathrm{X}(\mathrm{t})+\mathrm{hF}(\mathrm{t}, \mathrm{X}(\mathrm{t}), \mathrm{U}(\mathrm{t})), \mathrm{U}(\mathrm{t})+ \\
\left.\mathrm{h}\left[\lambda(\mathrm{t}) \mathrm{F}(\mathrm{t}, \mathrm{X}(\mathrm{t}), \mathrm{U}(\mathrm{t}))+\lambda^{\prime}(\mathrm{t}) \mathrm{X}(\mathrm{t})\right]\right) \\
\quad-\mathrm{V}(\mathrm{t}, \mathrm{X}(\mathrm{t}), \mathrm{U}(\mathrm{t})),
\end{gathered}
$$

using the Lipschitz condition give (i), thus we have $\mathrm{D}^{+} \mathrm{m}(\mathrm{t})=\lim _{\mathrm{h} \rightarrow 0^{+}} \sup \frac{1}{\mathrm{~h}}[\mathrm{~m}(\mathrm{t}+\mathrm{h})-\mathrm{m}(\mathrm{t})] \leq \mathrm{D}^{+} \mathrm{V}(\mathrm{t}, \mathrm{X}(\mathrm{t}), \mathrm{U}(\mathrm{t}))+$ $\lim _{h \rightarrow 0^{+}} \sup \frac{1}{h}\{D[X(t+h), X(t)+h F(t, X(t), U(t))]$ $-D\left[U(t+h), U(t)+h\left[\lambda(t) F(t, X(t), U(t))+\lambda^{\prime}(t) X(t)\right]\right\}$.

Since

$$
\frac{1}{h} \mathrm{D}[\mathrm{X}(\mathrm{t}+\mathrm{h}), \mathrm{X}(\mathrm{t})+\mathrm{hF}(\mathrm{t}, \mathrm{X}(\mathrm{t}), \mathrm{U}(\mathrm{t}))]=
$$

$$
\mathrm{D}\left[\frac{\mathrm{X}(\mathrm{t}+\mathrm{h})-\mathrm{X}(\mathrm{t})}{\mathrm{h}}, \mathrm{F}(\mathrm{t}, \mathrm{X}(\mathrm{t}), \mathrm{U}(\mathrm{t}))\right] \text {, }
$$

and $\mathrm{X}(\mathrm{t})$ is any solution of SCDEs (3), we find that

$$
\begin{gathered}
\lim _{\mathrm{h} \rightarrow 0^{+}} \sup \frac{1}{\mathrm{~h}}\{\mathrm{D}[\mathrm{X}(\mathrm{t}+\mathrm{h}), \mathrm{X}(\mathrm{t})+\mathrm{hF}(\mathrm{t}, \mathrm{X}(\mathrm{t}), \mathrm{U}(\mathrm{t}))]\} \\
=\mathrm{D}\left[\mathrm{D}_{\mathrm{H}} \mathrm{X}(\mathrm{t}), \mathrm{F}(\mathrm{t}, \mathrm{X}(\mathrm{t}), \mathrm{U}(\mathrm{t}))\right] \equiv 0,
\end{gathered}
$$

and 


$$
\begin{aligned}
& \lim _{h \rightarrow 0^{+}} \sup \frac{1}{h} D\left[U(t+h), U(t)+h\left[\lambda(t) F(t, X(t), U(t))+\lambda^{\prime}(t) X(t)\right]\right. \\
& =D\left[\lambda(t) D_{H} X(t)+\lambda^{\prime}(t) X(t), \lambda(t) F(t, X(t), U(t))+\lambda^{\prime}(t) X(t)\right] \\
& \leq \lambda(t)\left(D\left[D_{H} X(t), F(t, X(t), U(t))\right]+D\left[\lambda^{\prime}(t) X(t), \lambda^{\prime}(t) X(t)\right]\right) \equiv 0
\end{aligned}
$$

We therefore have the scalar differential inequality $D^{+} \mathrm{m}(\mathrm{t}) \leq \mathrm{g}(\mathrm{t}, \mathrm{m}(\mathrm{t})), \quad \mathrm{m}\left(\mathrm{t}_{0}\right) \leq \omega_{0}$, which yields, as before, the estimate $\mathrm{m}(\mathrm{t}) \leq \mathrm{r}\left(\mathrm{t}, \mathrm{t}_{0}, \omega_{0}\right)$, where $\mathrm{r}\left(\mathrm{t}, \mathrm{t}_{0}, \omega_{0}\right)$ is a maximal solution of ODE (5). This proof is complete.

Corollary 3.1. A function $\mathrm{g}(\mathrm{t}, \mathrm{m}(\mathrm{t})) \leq 0$ is admissible in the theorem 3.4 to yield the estimate

$$
\mathrm{V}(\mathrm{t}, \mathrm{X}(\mathrm{t}), \mathrm{U}(\mathrm{t})) \leq \mathrm{V}\left(\mathrm{t}_{0}, \mathrm{X}_{0}, \mathrm{U}_{0}\right), \quad \mathrm{t} \in\left[\mathrm{t}_{0}, \mathrm{~T}\right) .
$$

Next, we have some denotes:

$$
\begin{array}{r}
\mathrm{S}^{\mathrm{C}}(\rho)=\left\{\mathrm{X} \in \mathrm{K}_{\mathrm{C}}\left(\mathrm{R}^{\mathrm{n}}\right): \mathrm{D}\left[\mathrm{X}, \theta^{\mathrm{n}}\right]=\|\mathrm{X}\|<\rho\right\}, \\
\mathrm{K}=\left\{\sigma \in \mathrm{C}\left[\mathrm{R}_{+}, \mathrm{R}_{+}\right]: \sigma(\omega) \quad\right. \text { is increasing }
\end{array}
$$

$\omega$ and $\sigma(\omega) \rightarrow \infty$ as $\omega \rightarrow \infty\}$.

Now, we introduce some results on the boundedness of set solutions for SCDEs (3) by feedback $U(t)=\lambda(t) X(t)$.

Theorem 3.5. Assume that

$\mathrm{V} \in\left[\mathrm{R}_{+} \times \mathrm{K}_{\mathrm{C}}\left(\mathrm{R}^{\mathrm{n}}\right) \times \mathrm{U}, \mathrm{R}_{+}\right]$is Lyapunov-like function and $\mathrm{U}(\mathrm{t})=\lambda(\mathrm{t}) \mathrm{X}(\mathrm{t})$ is feedback for SCDEs (3) satisfies the following conditions:

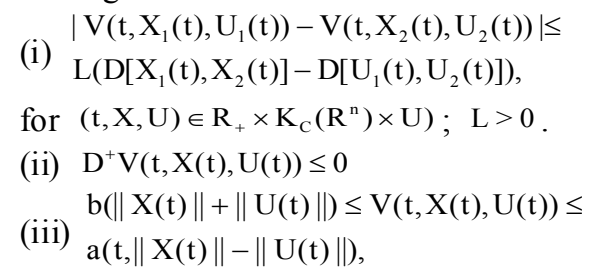

then, the affirmation (B1) holds.

Proof. Proof of this theorem is analogous proof of theorem 3.3.

Theorem 3.6. Assume that

(i) $\mathrm{V} \in\left[\mathrm{R}_{+} \times \mathrm{S}^{\mathrm{C}}(\rho) \times \mathrm{U}, \mathrm{R}_{+}\right]$, where $\rho$ may be large, satis fies:

$$
\begin{aligned}
& \left|\mathrm{V}\left(\mathrm{t}, \mathrm{X}_{1}(\mathrm{t}), \mathrm{U}_{1}(\mathrm{t})\right)-\mathrm{V}\left(\mathrm{t}, \mathrm{X}_{2}(\mathrm{t}), \mathrm{U}_{2}(\mathrm{t})\right)\right| \leq \\
& \mathrm{L}\left(\mathrm{D}\left[\mathrm{X}_{1}(\mathrm{t}), \mathrm{X}_{2}(\mathrm{t})\right]+\mathrm{D}\left[\mathrm{U}_{1}(\mathrm{t}), \mathrm{U}_{2}(\mathrm{t})\right]\right), \mathrm{L}>0 \\
& \text { (ii) for }(\mathrm{t}, \mathrm{X}(\mathrm{t}), \mathrm{U}(\mathrm{t})) \in \mathrm{R}_{+} \times \mathrm{S}^{\mathrm{C}}(\rho) \times \mathrm{U}, \\
& \mathrm{D}^{+} \mathrm{V}(\mathrm{t}, \mathrm{X}(\mathrm{t}), \mathrm{U}(\mathrm{t})) \leq 0 ; \\
& \text { (iii) } \mathrm{b}(\|\mathrm{X}(\mathrm{t})\|) \leq \mathrm{V}(\mathrm{t}, \mathrm{X}(\mathrm{t}), \mathrm{U}(\mathrm{t})) \leq \mathrm{a}(\|\mathrm{U}(\mathrm{t})\|) \\
& \text { and } 0<\lambda(\mathrm{t})<1 \text { where } \mathrm{a}(.), \mathrm{b}(.) \in \mathrm{K} \text {, which are defined }
\end{aligned}
$$
only on $[\rho, \infty)$,

then, (B2) holds.

Proof. We have to prove that (B2) holds. Because $\mathrm{g}(\mathrm{t}, \mathrm{V}(\mathrm{t}, \mathrm{X}(\mathrm{t}), \mathrm{U}(\mathrm{t})) \leq 0$ implies

$$
\begin{array}{r}
\mathrm{V}(\mathrm{t}, \mathrm{X}(\mathrm{t}), \mathrm{U}(\mathrm{t})) \leq \mathrm{V}\left(\mathrm{t}_{0}, \mathrm{X}\left(\mathrm{t}_{0}\right), \mathrm{U}\left(\mathrm{t}_{0}\right) \text { and } \forall \mathrm{t} \geq \mathrm{t}_{0}\right. \\
\mathrm{b}(\|\mathrm{X}(\mathrm{t})\|) \leq \mathrm{V}(\mathrm{t}, \mathrm{X}(\mathrm{t}), \mathrm{U}(\mathrm{t})) \leq \\
\mathrm{V}\left(\mathrm{t}_{0}, \mathrm{X}\left(\mathrm{t}_{0}\right), \mathrm{U}\left(\mathrm{t}_{\mathrm{o}}\right) \leq \mathrm{a}(\|\mathrm{X}(\mathrm{t})\|)\right.
\end{array}
$$

Thus for all $\mathrm{X}_{\mathrm{t}} \in \mathrm{S}^{\mathrm{C}}(\rho)$ and $\forall \mathrm{t}_{0} \in \mathrm{R}^{+}$there exists estimate $\mathrm{b}(\|\mathrm{U}(\mathrm{t})\|) \leq \mathrm{a}(\|\mathrm{X}(\mathrm{t})\|)$ then by (iii) of theorem 3.5 the affirmation for (B1) holds, that means (B2) holds.

\section{Conclusions}

By the Lyapunov like-funcions and by some kinds of feeback we just have investigated the problems of boundedness for set solutions to set control differential equations - SCDES, that is an one of the new trends in set-valued analysis. The boundedness properties of set solutions allows testing the ext re mal solutions, what is useful in practice of applications SDEs and SCDEs.

\section{ACKNOWLEDGEMENTS}

The authors gratefully acknowledge the referees for their careful reading and many valuable remarks which improved the presentation of the paper.

Thanks are due to the Vietnam National Foundation for Science and Technology Devolopment (NAFOSTED) for financial support.

\section{REFERENCES}

[1] T. Allahviranloo, A. Amirteimoori, M. Khezerloo, S. Khezerloo, "A New Method for Solving Fuzzy Volterra Integro-Differential Equations", Australian Journal of Basic and Applied Sciences, Vol. 5, Issue 4 (2011), pp 154 -164.

[2] Gnana Bhaskar T., Vasundhara Devi J. "Stability Criteria for Set Differential Equations", J. Mathematical and Computer Modelling, 2005, 41 (11-12): 1371-1378,doi:10.1016/j.mcm.2004. 01.012 .

[3] Gnana Bhaskar T., Vasundhara Devi J. "Nonuniform stability and boundedness criteria for set differential equations", J. Applicable Analy sis, 2005, 84 (2): 131-143, doi:10.1080/000 36810410001724346 .

[4] Hoa N.V., Phu N.D., "On maximal and minimal solutions for Set-valued Differential Equations with Feedback Control". Hindawi Publishing Corporation, J. Abstract and Applied Analysis, Volume 2012, Article ID 816218, 11 pages, doi: $10.1155 / 2012 / 816218$.

[5] Lakshmikantham V., Gnana Bhaskar T., Vasundhara Devi J., "Theory of set differential equations in metric spaces", Cambridge Scientific Publisher, UK, 2006.

[6] Lakshmikantham, V. and S. Leela, Fuzzy differential systems and the new concept of stability, J. Nonlinear Dynamics and Systems Theory, 1(1) (2001), pp 111--119.

[7] S. Li, A. Ren, "Representation theorems, set-valued and fuzzy set-valued Ito integral ", J. Fuzzy Sets and Systems, 158 (2007) pp 949-962.

[8] Michta, "On set-valued stochastic intergrals and fuzzy stochastic equations ", J. Fuzzy sets and Systems, (2011), doi: 10.1016/j.fss.2011.01.007.

[9] Nikolas S. Papageorgiou, "Existence of solutions for integrodifferential inclusions in Banach spaces", Comment.Math.U niv.Carolin, Vol. 32, Issue 4 (1991), pp 687 -696.

[10] Plotnikov A.V., Skripnik N.V., "Differential Equations with Set and Fuzzy Set-valued in Right hand side". OdessaUkraine, 2009. (In Russian) 192p. 
[11] Phu N.D., "On the Global Controllable for Set Control Differential Equations", International Journal of Evolution Equations, Vol. 4, Issue 3 (2009), pp 281 - 292.

[12] Phu N.D., Hoa N.V., Vu H., "On comparisons of set solutions for fuzzy control integro-differential systems", Journal of Advanced Research in Applied Mathematics, Vol. 4, Issue. 1, 2012, pp. 84-101, doi: 10.5373/jaram. 1081.08.2011

[13] Phu N. D., Quang L.T, Tung T.T., "Stability criteria for set control differential equations", J. Nonlinear Analysis: TMA, 69(2008), pp 3715 - 3721. doi:10.1016/j.na2006.07.01
[14] Phu N. D., Quang L. T. Hoa N.V.., "On the Existence of Extremal Solutions for Set Differential Equations" J. Advanced Research in Dynamical and Cotrol Systems, Vol 4 (2012), pp $18-28$.

[15] Phu N.D., Tung T.T., "On the Existence of fuzzy solutions of fuzzy control differential equations", J. Science and Technology Development 10, (5) (2006), pp 5--15.

[16] Phu N. D., Tung T.T., "Some results on sheaf solutions of sheaf set control differential equations" J. Nonlinear Analy sis: TMA,Vol 9 (2007), pp 1309 - 1315 